\title{
A SURPRISING HIGHER INTEGRABILITY PROPERTY OF MAPPINGS WITH POSITIVE DETERMINANT
}

\author{
STEFAN MÜLLER
}

Introduction. Let $\Omega$ be a bounded, open set in $\mathbf{R}^{n}, n \geq 2$, and assume that $u: \Omega \rightarrow \mathbf{R}^{n}$ belongs to the Sobolev space $W^{1, n}\left(\Omega ; \mathbf{R}^{n}\right)$, i.e. $\|u\|_{W^{1, n}}^{n}=\int_{\Omega}|u|^{n}+|D u|^{n} d x<\infty$, where $D u$ denotes the distributional derivative. Then det $D u$ is, of course, integrable. The aim of this note is to show that under the additional assumption that det $D u \geq 0$ (almost everywhere) in fact $\operatorname{det} D u \ln (2+\operatorname{det} D u)$ is integrable (on compact subsets $K$ of $\Omega$ ). When applied to a sequence of mappings $u^{j}: \Omega \rightarrow \mathbf{R}^{n}$ with $\operatorname{det} D u \geq 0,\left\|u^{(j)}\right\|_{W^{1, n}} \leq C$, this higher integrability result implies that the sequence $\operatorname{det} D u^{(j)}$ is weakly relatively compact in $L^{1}(K)$. This allows us to improve known results on weak continuity of determinants $[\mathbf{R}, \mathbf{B}]$ and existence of minimizers in nonlinear elasticity [BM]. In the terminology of Lions [L1, L2] and DiPerna and Majda [DM], the constraint $\operatorname{det} D u^{(j)} \geq 0$ prevents the development of 'concentrations' in the sequence $\operatorname{det} D u^{(j)}$.

One might ask whether analogous results hold for orientation preserving mappings between oriented compact Riemannian manifolds. In short, the function $\operatorname{det} D u \ln (2+\operatorname{det} D u)$ is still integrable, but not necessarily uniformly so along a sequence which is bounded in $W^{1, n}$. 'Concentrations' may occur, but only in a particular fashion (see $[\mathbf{M}]$ ).

THEOREM 1. Let $\Omega \subset \mathbf{R}^{n}$ be bounded and open and let $u: \Omega \rightarrow \mathbf{R}^{n}$ be in $W^{1, n}\left(\Omega ; \mathbf{R}^{n}\right), n \geq 2$. Assume that $\operatorname{det} D u \geq 0$ a.e. Then, for every compact set $K \subset \Omega, \operatorname{det} D u \ln (2+\operatorname{det} D u) \in L^{1}(K)$ and

$$
\|\operatorname{det} D u \ln (2+\operatorname{det} D u)\|_{L^{1}(K)} \leq C\left(K,\|u\|_{W^{1, n}(\Omega)}\right) \text {. }
$$

The result is optimal in the following sense. The assumption det $D u \geq 0$ cannot be dropped nor can $K$ be replaced by $\Omega$ (see Ball-Murat [BM, Counterexample 7.3]). Moreover det $D u \ln (2+\operatorname{det} D u)$ cannot be replaced by $\gamma(\operatorname{det} D u)$ with $\gamma(z) /(z \ln (2+z)) \rightarrow+\infty$ for $z \rightarrow+\infty$ (see [M]).

Two key lemmas. The proof of Theorem 1 relies on a geometric estimate (a version of the isoperimetric inequality) and an analytic result on maximal functions by Stein [S2]. We begin with the former. For an $n \times n$ matrix $F$ let adj $F$ denote the transpose of the matrix of cofactors, so that $F \operatorname{adj} F=\operatorname{det} F$ Id.

LemMA 2. Let $\Omega \subset \mathbf{R}^{n}$ be bounded and open and let $u \in W^{1, n}\left(\Omega ; \mathbf{R}^{n}\right)$. For $x \in \Omega$ let $B_{d}(x)$ be a ball of radius $d$ around $x$ such that $B_{d}(x) \subset \Omega$.

Received by the editors May 8, 1989.

1980 Mathematics Subject Classification (1985 Revision). Primary 49A22, 58C25; Secondary $26 \mathrm{~B} 35,73 \mathrm{C} 50$. 
Then, for a.e. $r \in(0, d)$,

$$
\left|\int_{B_{r}(x)} \operatorname{det} D u d y\right|^{(n-1) / n} \leq c \int_{\partial B_{r}(x)}|\operatorname{adj} D u| d S,
$$

where the constant $c$ depends only on $n$.

If $u$ is a $C^{1}$-diffeomorphism, (2) follows from the usual isoperimetric inequality as the left-hand side is $\left\{\operatorname{vol} u\left(B_{r}\right)\right\}^{(n-1) / n}$ while the right-hand side is an upper bound for area $u\left(\partial B_{r}\right)$ times a constant. As stated, Lemma 2 is an immediate consequence of the isoperimetric inequality for currents (see Federer [F, Theorem 4.5.9 (31)]); an elementary proof, based on approximation by smooth functions and degree theory is also available.

Recall that for $f \in L^{1}\left(\mathbf{R}^{n}\right)$ the maximal function $M f$ is defined by

$$
M f(x)=\sup _{R>0} \frac{1}{\operatorname{meas} B_{R}(x)} \int_{B_{R}(x)}|f(y)| d y .
$$

Lemma 3 (STEIN [S2]). Let $f \in L^{1}\left(\mathbf{R}^{n}\right)$ and assume that $f$ is supported on $a$ ball $B$ and that $M f \in L^{1}(B)$. Then $|f| \ln (2+|f|) \in L^{1}(B)$ and

$$
\||f| \ln (2+|f|)\|_{L^{1}(B)} \leq C\left(B,\|M f\|_{L^{1}(B)}\right) .
$$

Estimate (3) is implicit in [S1, p. 23,S2], though not explicitly stated.

Proof of Theorem 1. Fix $K \subset \Omega$, compact and let

$$
g=1_{K} \operatorname{det} D u,
$$

$1_{K}$ being the characteristic function of $K$. By Lemma 3 we only have to show that the maximal function $M g$ satisfies

$$
\|M g\|_{L^{1}(B)} \leq C\left(K,\|u\|_{W^{1, n}(\Omega)}\right),
$$

for some ball $B \supset \Omega$. Let $d=\operatorname{dist}(K, \partial \Omega)$. It suffices to estimate

$$
\frac{1}{\operatorname{meas} B_{R}(x)} \int_{B_{R}(x)}|g(y)| d y,
$$

for $x$ satisfying $\operatorname{dist}(x, \partial \Omega)>d / 2$ and for $R<d / 4$, as otherwise (5) is bounded by $C(d)\|u\|_{W^{1, n}(\Omega)}$.

Using the fact that det $D u \geq 0$ and Lemma 2 we have, for a.e. $r \in$ $(R, 2 R)$,

$$
\begin{aligned}
\left\{\int_{B_{R}(x)}|g(y)| d y\right\}^{(n-1) / n} & \\
& \leq\left\{\int_{B_{r}(x)} \operatorname{det} D u d y\right\}^{(n-1) / n} \leq c \int_{\partial B_{r}(x)}|\operatorname{adj} D u| d S .
\end{aligned}
$$

Here and in the following we denote by $c$ any constant depending solely on $n$. Integrating the above inequality over $r$ from $R$ to $2 R$ and dividing 
by $R^{n}$ we obtain

$$
\begin{aligned}
\left\{\frac{1}{\operatorname{meas} B_{R}(x)} \int_{B_{R}(x)}|g(y)| d y\right\}^{(n-1) / n} & \\
& \leq \frac{c}{\operatorname{meas} B_{2 R}(x)} \int_{R_{2 R}(x)}|\operatorname{adj} D u| d y \leq c M f,
\end{aligned}
$$

where $M f$ is the maximal function of $f=1_{\Omega}|\operatorname{adj} D u|$. Thus

$$
M g(x) \leq c\{M f(x)\}^{n /(n-1)}+C(d)\|u\|_{W^{1, n}}^{n} .
$$

Now $f \in L^{n /(n-1)}$, and hence [S1, I, Theorem 1]

$$
\|M f\|_{L^{n /(n-1)}} \leq c\|f\|_{L^{n /(n-1)}} \leq c\|u\|_{W^{1, n(\Omega)}}
$$

so that (4) follows.

Applications. Theorem 1 allows to sharpen previous results by Reshetnyak [R] and Ball [B] on the weak continuity of determinants.

Corollary 4. Let $\Omega \subset \mathbf{R}^{n}$ be open and bounded, and assume that the sequence of mappings $u^{(j)}: \Omega \rightarrow \mathbf{R}^{n}$ satisfies $\operatorname{det} D u^{(j)} \geq 0$ and $u^{(j)} \rightarrow u$ (weakly) in $W^{1, n}\left(\Omega ; \mathbf{R}^{n}\right)$. Then

$$
\operatorname{det} D u^{(j)} \rightarrow \operatorname{det} D u\left(\text { weakly) in } L^{1}(K),\right.
$$

for all compact sets $K \subset \Omega$.

In [R, B] it is shown that $\operatorname{det} D u^{(j)} \rightarrow \operatorname{det} D u$ weak* in the sense of measures. Since $\left\|u^{(j)}\right\|_{W^{1, n}} \leq C$, Theorem 1 in combination with the criterion on weak compactness in $L^{1}$ (see [ET, VIII, Theorem 1.3]) implies that the sequence det $D u^{(j)}$ is weakly relatively compact in $L^{1}(K)$, and (6) follows. Corollary 4 , but not Theorem 1 , can also be deduced from a recent result by Zhang [Z]. In [M] Corollary 4 is used to improve a result of Ball and Murat [BM, Theorem 6.1] on the existence of minimizers in nonlinear elasticity. Both Theorem 1 and Corollary 4 should also have interesting applications in geometry.

Acknowledgements. I wish to thank J. M. Ball, M. Esteban, F. Murat and $\mathrm{K}$. W. Zhang for very fruitful discussions.

Note ADDED IN PROOF. Since this paper was submitted, Theorem 1 has let to several interesting developments. R. Coifman, Y. Meyer, P. L. Lions and S. Semmes found a new proof based on 'hard' harmonic analysis. Assuming only $u \in W^{1, n}$ they show first that det $D u$ is in the Hardy space $\mathscr{H}^{1}$ (the predual of BMO). A standard result (similar to Lemma 3 ) then states that a positive function is in $\mathscr{H}^{1}$ if and only if $f \ln (2+f)$ is integrable. Their proof uses directly the divergence structure of the determinant rather than geometric estimates such as the isoperimetric inequality and thus has potential applications to more general situations. 


\section{REFERENCES}

[B] J. M. Ball, Convexity conditions and existence theorems in nonlinear elasticity, Arch. Rational Mech. Anal. 63 (1977), 337-403.

[BM] J. M. Ball and F. Murat, $W^{1, p}$-quasiconvexity and variational problems for multiple integrals, J. Funct. Anal. 58 (1984), 225-253.

[DM] R. J. DiPerna and A. J. Majda, Oscillations and concentrations in weak solutions of the incompressible fluid equations, Comm. Math. Phys. 108 (1987), 667-689.

[ET] I. Ekeland and R. Teman, Convex analysis and variational problems, North-Holland, Amsterdam, 1976.

[F] H. Federer, Geometric measure theory, Springer-Verlag, Berlin, Heidelberg, New York, 1969.

[L1] P. L. Lions, The concentration-compactness principle in the calculus of variations, the locally compact case, parts I and II, Ann. Inst. H. Poincaré 1 (1984), 109-145; 223-283.

[L2] _ - The concentration compactness principle in the calculus of variations, the limit case, part I, Rev. Mat. Iberoamericana 1 (1984), 145-201; part II 2 (1985), 45-121.

[M] S. Müller, Higher integrability of determinants and weak compactness in $L^{1}$, preprint.

[R] Y. G. Reshetnyak, Stability theorems for mappings with bounded excursion, Siberian Math. J. 9 (1968), 499-512.

[S1] E. Stein, Singular integrals and differentiability properties of functions, Princeton Univ. Press, Princeton, N. J., 1970.

[S2] _ Note on the class L $\log L$, Studia Math. 32 (1969), 305-310.

[Z] K. W. Zhang, Biting theorems for jacobians and their applications, preprint.

Department of Mathematics, Heriot-Watt University, Edinburgh EH14 4AS, SCOTLAND, UNITED KINGDOM 